\title{
Midwifery empathy scale: development and validation for a greek sample
}

\author{
Victoria G. Vivilaki ${ }^{*}$, Polyxeni Fifli', Anastasia Charitou', Paraskevi Giaxi ${ }^{1}$, Chrysoula Ekizoglou', \\ Nicholas D. Tsopelas ${ }^{2}$, Maria Bouroutzoglou ${ }^{3}$ and Evridiki Patelarou ${ }^{4,5}$
}

\begin{abstract}
Background: In recent years, a growing number of studies report on the importance of empathy to women's care. The Midwifery Empathy Scale (M.E.S) was developed in order to study and record the levels of empathy of professional and student midwives. The purpose of our study was to validate this instrument and determine the factor structure and reliability.

Methods: The study sample $(n=242)$ consisted of professional $(n=114)$ and student midwives $(n=128)$ of 3 Maternity Hospitals (public and private) of Athens.

Results: The exploratory factor analysis on the 25 item of Midwifery Empathy Scale (MES) revealed seven orthogonal factors (KMO Measure of Sample Adequacy $=0.817$ and Bartlett's test of sphericity $=1508.169, \mathrm{df}=300$, $p<0.0001$. The MES showed medium overall internal consistency (Cronbach's alpha value: $0.546, p<0.0001$ ). The internal consistency characteristics of MES demonstrated good reliability. Our findings attest to the multidimensionality of MES, and the Confirmatory Factor Analysis (CFA) demonstrated that the 7 factor model offers a very good fit to our data.
\end{abstract}

Conclusion: Our data indicate that the Midwifery Empathy Scale (MES) is a reliable and valid tool for assessing the levels of empathy of professional and student midwives for both clinical and research practices.

Keywords: Midwives, Compassionate, Empathetic, Emotional detachment, Perspective taking, Interpersonal relationship, Tendencies

\section{Background}

Empathy is considered a key parameter in compassionate care and understanding its nature can be of great significance for women's care [1-3] and for the midwives [4-7].

Studying the levels of empathy through a practical psychometric tool it is expected to improve the quality of the midwifery care. There are a few studies in the relevant literature exploring the levels of empathy in student midwives [8], and professional midwives $[9,10]$. At the same time, there was no validated instrument designed to assess the levels of empathy specifically for midwives. The Midwifery Empathy Scale (MES), a 25- item psychometric scale, was developed to meet this challenge.

\footnotetext{
* Correspondence: v_vivilaki@yahoo.co.uk

${ }^{1}$ Department of Midwifery, Technological Educational Institute of Athens,

Athens, Greece

Full list of author information is available at the end of the article
}

Therefore, the aim of the present study was to develop and determine the factor structure, validity and reliability of the MES by measuring student and professional midwives' levels of empathy.

\section{Methods \\ Pilot study}

The 25-item MES was developed after extensive literature review, targeting only midwives. The midwifery underpinning philosophy, culture and clinical practice was taken into consideration. The scale was originally tested in a small pilot study with 7 midwives. In the process of cultural adaptation, we conducted in depth interviews to examine respondents' understanding of the scale items. Participants provided their views about the clarity of each item, the relevance of the content to their situation, the comprehensiveness of the instructions and their ability to complete the scale on their own. 


\section{Data collection}

The research was approved by the Ethical Committees of the participant Hospitals (No51/27-2-2015 \& No17/ 24-2-2015) and validation activities were initiated in the period of March to June 2015. Following written informed consent, students and professional midwives completed the scale. A cover letter explained the purpose of the study, provided the researchers' affiliation and contact information and guaranteed confidentiality and anonymity.

\section{Participants}

Student midwives $(n=128)$ and professional midwives $(n=114)$ were recruited from 3 Hospitals of Athens municipality (public and private) and the midwifery Department of TEI of Athens. The inclusion criteria required fluency in spoken and written Greek language, being a student or a professional midwife and written informed consent.

\section{Instrument}

The MES is a 25-item scale that consists of situations where empathetic responses are evaluated. Each item is scored on a 6-point Likert scale from 1-6 with response options that range from 1 (totally agree) to 6 (totally disagree). A total score for MES is calculated (25-150). Items that measure negative statements are reversely scored with lower scores indicating lower levels of empathy.

\section{Data analysis}

Statistical analysis was performed using IBM SPSS statistics version 20. Descriptive characteristics (including means, standard deviation, frequencies and percentages) were calculated for the socio-demographic variables.

\section{Reliability}

Reliability coefficients, measured by Cronbach's alpha, were calculated for MES to assess reproducibility and consistency of the instrument; a minimum value of 0.70 for group comparisons is considered acceptable [11].

\section{Factor structure}

The underlying dimensions of the scale were checked with an exploratory factor analysis using a Varimax rotation and Principal Components Method for analyzing group data [12] to determine the dimensional structure of MES using the following criteria: (a) eigenvalue $>1$ [13], (b) variables loaded $>0.50$ on only one factor and on other factors less than 0.40; (c) the interpretation of the factor structure was meaningful, (d) the screenplot was accurate when means of communalities were above 0.60 [14]. Computations were based on a measurement scale [15]. During factor analysis, a Barlett's test of sphericity $(p<0.05)$ and a Kaiser-Meyer-Olkin (KMO) measure of sampling adequacy of 0.817 were also implemented. A factor was considered as important if its eigenvalue exceeded 1.0 [16]. Additionally, a confirmatory analysis -also called Structural Equation Modelling- of principal components was conducted by STATA 13.1 to confirm the scale items principally load on to that factor and correlate weakly with other factors. To assess tests for significance of factor loadings and orthogonality of factors $[12,14,16]$ a model (based on a priori information of exploratory factor analysis)- was built in order to specify latent factors, their component variables and the intercorrelations of the response variables; maximum likelihood estimates, t-values, error terms, correlation of independent variables and goodness of fit-test for the specified model were performed.

\section{Results}

During the recruitment period (March 2015-June 2015), 300 eligible student and professional midwives were invited to participate. Fifty eight of them did not participate for a range of reasons (19 refused, 24 missing values, 8 missed, 7 other). This resulted in a final sample of 242 student and professional midwives which was adequate for exploratory factor analysis [17-19]. The questionnaire response rate was $80,6 \%$.

\section{Sample characteristics}

According to the answers to questions, 234 from 242 respondents are women. The age of the midwives ranges from 19-58 (mean 28.68). Most of them (47.1\%) were graduates from direct entry midwifery school (TEI), while fewer $(11.2 \%)$ has post-graduate studies and $0.81 \%$ had a $\mathrm{PhD} ; 52.1 \%$ were student midwives practicing in the public hospitals; $24.8 \%$ of them were married (Table 1 ).

\section{Psychometric characteristics of MES Reliability}

The MES showed an overall medium consistency. Cronbach's alpha was 0,546 $p<0,0001$ for the total scale (Items 1-25).

\section{Factor structure Exploratory factor}

The exploratory factor analysis of the 25 items of the MES revealed seven orthogonal factors (KMO measure of sampling adequacy $=0.817$ and Barlett's test of sphericity $=1508.169 \mathrm{df}=300 p<0.0001)$. Communalities for MES questions are presented in (Table 2). The Screeplot (Fig. 1) and Component Plot in Rotated Space (Fig. 2) that are 7 factors in the model, with these factors explaining 57,065 \% of the data (Table 3). The first factor (Compassionate Care) includes the following items: $6,12,13,15,20$, the eigenvalue was 6.158 and accounted 
Table 1 Characteristics of the Study Sample

\begin{tabular}{ll}
\hline & All participants No (\%) \\
\hline Sex & $7(2.9 \%)$ \\
Male & $234(96.7 \%)$ \\
Female & \\
Public & $71(29.3 \%)$ \\
Private & $33(13.6 \%)$ \\
Marital Status & \\
Single & $175(72.3 \%)$ \\
Married & $60(24.8 \%)$ \\
Divorced & $6(2.5 \%)$ \\
Education & \\
Student & $126(52.1 \%)$ \\
TEl & $114(47.1 \%)$ \\
Postgraduate & $18(7.4 \%)$ \\
Doctoral & $2(0.8 \%)$ \\
\hline
\end{tabular}

$24.632 \%$ of the variance. The second factor (Empathetic Ways) includes the items : 14,18,21,24, the eigenvalue was 1.792 and accounted for $7.169 \%$ of the variance. The third factor (Emotional Detachment) is composed of items: 10, 19, 25, the eigenvalue was 1.443 and accounted for $5.771 \%$ of the variance. The fourth factor (Perspective Taking) is composed of items: 1, 3, 7 had an eigenvalue 1.366 and accounted for $5.464 \%$ of the variance. The fifth factor (Interpersonal Relationship) includes the items: 22, 23 had an eigenvalue of 1.287 and accounted for an additional $5.149 \%$ of the variance. The sixth factor (Standing in the woman's shoes) includes the following items: 8,11 had an eigenvalue of 1.205 and accounted for an additional $4.821 \%$ of the variance. Finally, the seventh factor (Empathetic Tendencies) consists items: 9, 16, 17 with an eigenvalue of 1.015 and accounted for an additional $4.059 \%$ of the variance (Table 3).

According to MES validation, 3 out of the 25 items, which were not relevant for the Greek midwives (Items: $2,4,5)$.

Table 2 Descriptive Statistics of the MES items

\begin{tabular}{|c|c|c|}
\hline Question & Mean & Std. Deviation \\
\hline 1. I believe that empathy plays an important role in midwifery care. & 1,46 & 683 \\
\hline 2. Midwives should understand the emotional situation of the women and their families. & 1,45 &, 590 \\
\hline 3. I can perceive the hidden feelings and thoughts of the women that are in my care. & 2,42 & ,796 \\
\hline 4. During the taking of the medical history it is not important to pay attention to the women's feelings. & 5,04 & 1,276 \\
\hline 5. Women's emotions do not concern me. & 5,55 & 853 \\
\hline 6. Women feel better when they sense that they are understood. & 1,32 & 603 \\
\hline 7. I recognize the body language of a woman. & 2,28 & ,763 \\
\hline 8. Body language is not as important as verbal communication for the understanding of the woman's feelings. & 4,52 & 1,300 \\
\hline 9. I recognize when a woman is silent because of embarrassment. & 2,07 & ,693 \\
\hline 10. I don't get emotionally affected when I see women cry. & 4,34 & 1,369 \\
\hline 11. It is difficult for a midwife to see things from women's perspective. & 4,75 & 1,199 \\
\hline 12. I try to stand in the woman's shoes, so I can better understand her. & 1,99 & 991 \\
\hline 13. I show that I am willing to listen to the woman by always sitting near her. & 1,70 &, 774 \\
\hline 14. I would spend time to take care of women after my work hours. & 2,54 & 1,271 \\
\hline 15. Midwife's touch encourages the woman. & 1,66 & ,742 \\
\hline 16. I avoid to touch the woman I am caring for, in order to keep a distance. & 5,05 & 886 \\
\hline 17. I think it is important to touch a woman when I am caring for her. & 1,90 & 840 \\
\hline 18. Very sensitive women irritate me. & 4,58 & 1,211 \\
\hline 19. There were times that I witnessed a woman cry and I got emotional. & 2,32 & 1,131 \\
\hline 20. Many times I left work and I kept thinking of a woman I was caring for. & 1,90 & 960 \\
\hline 21. I don't think part of my job to occupy myself with the problems of the woman I care. & 4,85 & 1,188 \\
\hline 22. I feel satisfaction when women feel better with my care. & 1,31 & ,704 \\
\hline 23. If I realize that a woman is afraid, I spend time trying to reassure her. & 1,58 & ,743 \\
\hline 24. I could go against hospital rules in order to help a woman. & 3,14 & 1,304 \\
\hline 25. I usually stay emotionally detached from the women that are in my care. & 4,26 & 1,322 \\
\hline
\end{tabular}




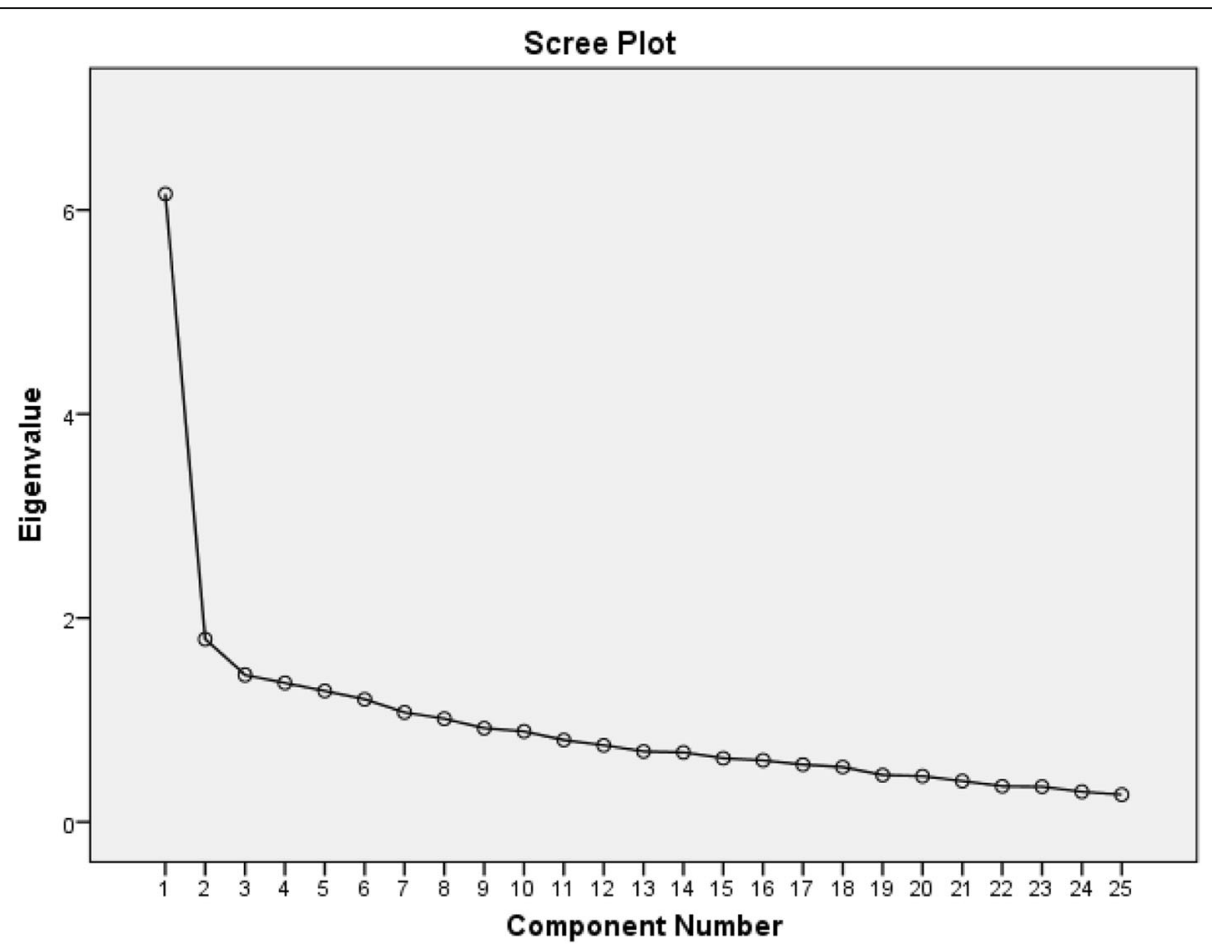

Fig. 1 Screeplot

\section{Component Plot in Rotated Space}

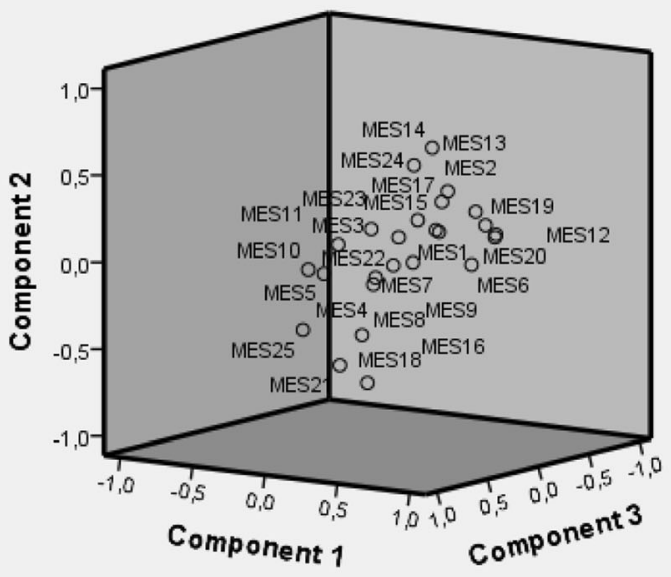

Fig. 2 Component Plot in Rotated Space 
Table 3 Exploratory factors and Explained Variance after rotation for MES

\begin{tabular}{|c|c|c|c|c|c|c|c|}
\hline \multirow[t]{2}{*}{ Factors } & & \multirow{2}{*}{$\begin{array}{l}\text { Rescaled } \\
\text { Loadings }\end{array}$} & \multirow{2}{*}{$\begin{array}{l}\text { Eigen } \\
\text { values }\end{array}$} & \multicolumn{4}{|c|}{ Rotation Sums of Squared Loadings } \\
\hline & & & & $\%$ of Variance & Cumulative Variance & Cronbach's alpha & Standardised alpha \\
\hline \multirow[t]{5}{*}{ Factor 1 (Compassionate Care) } & Q6 & 0.626 & 6.158 & 24.632 & 24.632 & 0.720 & 0.731 \\
\hline & Q12 & 0.719 & & & & & \\
\hline & Q13 & 0.648 & & & & & \\
\hline & Q15 & 0.469 & & & & & \\
\hline & Q20 & 0.534 & & & & & \\
\hline \multirow[t]{4}{*}{ Factor 2 (Empathetic Ways) } & Q14 & 0.640 & 1.792 & 7.169 & 31.801 & 0.564 & 0.577 \\
\hline & Q18 & 0.739 & & & & & \\
\hline & Q21 & 0.620 & & & & & \\
\hline & Q24 & 0.533 & & & & & \\
\hline \multirow[t]{3}{*}{ Factor 3 (Emotional Detachment) } & Q10 & 0.735 & 1.443 & 5.771 & 37.572 & 0.465 & 0.505 \\
\hline & Q19 & 0.709 & & & & & \\
\hline & Q25 & 0.650 & & & & & \\
\hline \multirow[t]{3}{*}{ Factor 4 (Perspective Taking) } & Q1 & 0.489 & 1.366 & 5.464 & 43.036 & 0.592 & 0.588 \\
\hline & Q3 & 0.787 & & & & & \\
\hline & Q7 & 0.756 & & & & & \\
\hline \multirow[t]{2}{*}{ Factor 5 (Interpersonal Relationship) } & Q22 & 0.895 & 1.287 & 5.149 & 48.185 & 0.766 & 0.767 \\
\hline & Q23 & 0.771 & & & & & \\
\hline \multirow[t]{2}{*}{ Factor 6 (Standing in the woman's shoes) } & Q8 & 0.753 & 1.205 & 4.821 & 53.006 & 0.514 & 0.515 \\
\hline & Q11 & 0.682 & & & & & \\
\hline \multirow[t]{3}{*}{ Factor 7 (Empathetic Tendencies) } & Q9 & 0.720 & 1.015 & 4.059 & 57.065 & 0.572 & 0.556 \\
\hline & Q16 & 0.511 & & & & & \\
\hline & Q17 & 0.488 & & & & & \\
\hline
\end{tabular}

\section{Confirmatory factor analysis}

Confirmatory factor analysis was conducted to determine whether data are consistent with the a priori specified model that has been suggested by Exploratory Factor Analysis in order to evaluate whether the data fit the model adequately. The seven factor-model was based on correlated factors that derived from the factor analysis using principal component analysis with varimax rotation by SPSS 20. The seven latent variables Compasionate Care (Questions 6, 12, 13, 15, 20), Empathetic Ways (Questions 14, 18, 21, 24), Emotional Detachment (Questions 10, 19, 25), Perspective Taking (Questions 1, 3, 7), Interpersonal Relationship (Questions 22, 23), Standing in the woman's shoes (Questions 8,11) and Empathetic Tendencies (Questions 9, 16, 17) were strongly correlated with method Maximum Likelihood. Estimates, standard error, $t$-values, error terms and $r 2$ for all the questions that consisted each latent variables are presented at Fig. 3. The error terms correlated significantly and Goodness of Fit Statistics were also estimated; Discrepancy Chi-Square $=2340.841, p=0.000$; Standardized Root Mean Square Residual $(\mathrm{SRMR})=0.323 ; \mathrm{CD}=1.000$ (Fig. 4).

\section{Validity}

Construct validity

Cronbach's alpha was calculated for each of the following subscales of the MES, with the questions indicated constituting the items of each subscale:

1. Compassionate Care $(6,12,13,15,20): 0.731$

2. Empathetic Ways $(14,18,21,24): 0.577$

3. Emotional Detachment (10,19,25): 0.405

4. Perspective Taking (1,3,7): 0.592

5. Interpersonal Relationship (22, 23): 0.767

6. Standing in the woman's shoes (8,11): 0.515

7. Empathetic Tendencies $(9,16,17): 0.572$

\section{Discussion}

Main findings

The MES is a scale designed to measure the empathy of professional and student midwives. It was developed to meet the challenge of developing a psychometric tool tailored to assess the levels of empathy for midwives. The results of this research showed that the major formative factors of the empathy levels in midwives are: 1) Compassionate Care 2) Empathetic Ways 3) Emotional 


\begin{tabular}{|c|c|c|c|c|c|c|c|}
\hline \multirow{3}{*}{$\begin{array}{l}\text { Measurement } \\
\text { MESG <- }\end{array}$} & \multirow{3}{*}{$\begin{array}{c}\text { Standardized } \\
\begin{array}{r}\text { } \\
\text { compassionate } \\
\text { cons }\end{array} \\
\end{array}$} & \multirow{3}{*}{ 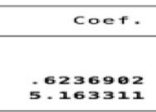 } & \multirow{3}{*}{$\begin{array}{l}\text { Std. Err. } \\
.1374743 \\
: 9297113\end{array}$} & \multirow{3}{*}{ 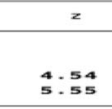 } & \multirow{3}{*}{ 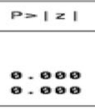 } & \multirow{3}{*}{$\begin{array}{l}195 \% \text { conf. } \\
\begin{array}{l}3542455 \\
3.3411\end{array}\end{array}$} & \multirow{3}{*}{$\begin{array}{l}\text { Intervatu } \\
\text { 8931349 } \\
6.985512 \\
\end{array}$} \\
\hline & & & & & & & \\
\hline & & & & & & & \\
\hline Mesiz <- & $\begin{array}{c}\text { Compassionate } \\
\text {-cons }\end{array}$ & $\begin{array}{r}5591341 \\
2.257295\end{array}$ & $\begin{array}{l}0527031 \\
0663475\end{array}$ & $\begin{array}{l}10.6 x \\
34: 02\end{array}$ & 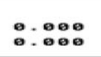 & $\begin{array}{r}4558379 \\
2.127256\end{array}$ & $\begin{array}{r}6624302 \\
2.338333\end{array}$ \\
\hline $\operatorname{mes} 13<-$ & $\begin{array}{r}\text { compassionate } \\
\text {-conss }\end{array}$ & $\begin{array}{r}8602767 \\
2.310589\end{array}$ & $\begin{array}{l}0400724 \\
.0637798\end{array}$ & $\begin{array}{l}21: 47 \\
36: 23\end{array}$ & 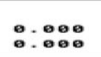 & $\begin{array}{r}7817361 \\
2.185583\end{array}$ & $\begin{array}{r}-9388172 \\
2.435595\end{array}$ \\
\hline MES15 <- & $\begin{array}{r}\text { Compassionate } \\
\text {-cons }\end{array}$ & $\begin{array}{r}756096 \\
2.561406 \\
\end{array}$ & $\begin{array}{r}0487516 \\
-6623044 \\
\end{array}$ & $\begin{array}{l}15.51 \\
41: 11 \\
\end{array}$ & 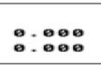 & $\begin{array}{r}6605446 \\
2.439291 \\
\end{array}$ & $\begin{array}{r}8516474 \\
2.68352 \\
\end{array}$ \\
\hline MESZO <- & $\begin{array}{r}\text { compassionate } \\
\text {-conns }\end{array}$ & $\begin{array}{r}5661248 \\
3.361086\end{array}$ & $\begin{array}{r}0590628 \\
2133126\end{array}$ & $\begin{array}{r}9.59 \\
15: 76\end{array}$ & 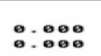 & $\begin{array}{r}4503637 \\
2.943001\end{array}$ & $\begin{array}{r}-6818859 \\
3.779172\end{array}$ \\
\hline MES14<- & $\begin{array}{r}\text { Empatheticw } \\
\text {-coons }\end{array}$ & $\begin{array}{r}8280714 \\
2.05981 \\
\end{array}$ & $\begin{array}{r}0246459 \\
0545016\end{array}$ & $\begin{array}{r}33.60 \\
37: 70\end{array}$ & \begin{tabular}{l}
0.00 \\
\hdashline$: \infty 800$
\end{tabular} & $\begin{array}{r}7797663 \\
i .952988\end{array}$ & $\begin{array}{r}8763765 \\
2.166631 \\
\end{array}$ \\
\hline MESIA <- & $\begin{array}{r}\text { Empatheticw } \\
\text {-cons }\end{array}$ & $\begin{array}{r}6969914 \\
-747793\end{array}$ & $\begin{array}{l}0352342 \\
16674632 \\
\end{array}$ & $\begin{array}{r}-19.78 \\
28.35\end{array}$ & 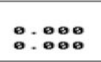 & $\begin{array}{r}-7660492 \\
4.419571\end{array}$ & $\begin{array}{r}-.6279336 \\
5.076015\end{array}$ \\
\hline MESZ1 <- & $\begin{array}{r}\text { Empatheticw } \\
- \text { cons }\end{array}$ & $\begin{array}{r}-804669 \\
-80429898\end{array}$ & $\begin{array}{r}0410928 \\
1953687 \\
\end{array}$ & $\begin{array}{r}-19.58 \\
27: 28 \\
\end{array}$ & \begin{tabular}{r}
0.00 \\
\hdashline$: 000$ \\
\end{tabular} & $\begin{array}{r}-.8852972 \\
4.946999\end{array}$ & $\begin{array}{r}-.7241265 \\
5.712798\end{array}$ \\
\hline MESZ4 <- & $\begin{array}{r}\text { Empatheticw } \\
\text {-cons }\end{array}$ & $\begin{array}{r}6700584 \\
3.094 \\
\end{array}$ & $\begin{array}{r}0320962 \\
: 1215688 \\
\end{array}$ & $\begin{array}{r}20 \cdot 88 \\
24: 71 \\
\end{array}$ & 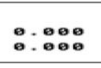 & $\begin{array}{r}607151 \\
2.765745 \\
\end{array}$ & $\begin{array}{r}7329657 \\
3.242255 \\
\end{array}$ \\
\hline MES10 <- & $\begin{array}{c}\text { Emotional } \\
\text {-cons }\end{array}$ & $\begin{array}{r}5056432 \\
3.986697\end{array}$ & $\begin{array}{r}040723 \\
.1359146 \\
\end{array}$ & $\begin{array}{l}12.42 \\
29.29\end{array}$ & 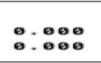 & $\begin{array}{r}4258275 \\
3.714369\end{array}$ & $\begin{array}{r}5854589 \\
4.247085 \\
\end{array}$ \\
\hline MEsis <- & $\begin{array}{r}\text { Emotianal } \\
\text {-cons } \\
\end{array}$ & $\begin{array}{l}-597813 \\
2: 781961 \\
\end{array}$ & $\begin{array}{r}0726418 \\
-1279525 \\
\end{array}$ & $\begin{array}{l}-8: 23 \\
21: 74\end{array}$ & \begin{tabular}{r}
$\mathbf{0}-\infty$ \\
\hdashline$-\infty$ \\
\end{tabular} & $\begin{array}{r}-7401884 \\
2.531118\end{array}$ & $\begin{array}{r}-.4554377 \\
3.032683 \\
\end{array}$ \\
\hline MES25 <- & $\begin{array}{c}\text { Emotional } \\
\text {-cons }\end{array}$ & $\begin{array}{r}6016594 \\
4.144662 \\
\end{array}$ & $\begin{array}{r}045453 \\
.1279416 \\
\end{array}$ & $\begin{array}{l}13.24 \\
32: 39 \\
\end{array}$ & 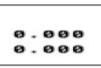 & $\begin{array}{r}5125731 \\
3.893901 \\
\end{array}$ & $\begin{array}{r}6907456 \\
4.395423 \\
\end{array}$ \\
\hline MESI <- & $\begin{array}{r}\text { Perspective } \\
\text {-cons }\end{array}$ & $\begin{array}{r}5223508 \\
2.576413 \\
\end{array}$ & $\begin{array}{r}0469104 \\
-1203555 \\
\end{array}$ & $\begin{array}{l}11: 14 \\
21: 41 \\
\end{array}$ & \begin{tabular}{r}
$0: 00$ \\
\hdashline$: \infty 80$ \\
\end{tabular} & $\begin{array}{r}4304082 \\
2.340521 \\
\end{array}$ & $\begin{array}{r}6142934 \\
2.812306 \\
\end{array}$ \\
\hline MES3 $<-$ & $\begin{array}{r}\text { Perspective } \\
\text {-cons }\end{array}$ & $\begin{array}{r}731517 \\
3.814209 \\
\end{array}$ & $\begin{array}{r}0597058 \\
1453904 \\
\end{array}$ & $\begin{array}{l}12.25 \\
26.23 \\
\end{array}$ & \begin{tabular}{r} 
: : \\
\hdashline$: 00$ \\
\end{tabular} & $\begin{array}{r}6144959 \\
3.529249 \\
\end{array}$ & $\begin{array}{r}8485382 \\
4.099169 \\
\end{array}$ \\
\hline MEST <- & $\begin{array}{r}\text { Perspectuve } \\
\text {-cons }\end{array}$ & $\begin{array}{r}7138211 \\
3.261893 \\
\end{array}$ & $\begin{array}{r}0456058 \\
1117159 \\
\end{array}$ & $\begin{array}{l}15: 32 \\
29: 20 \\
\end{array}$ & \begin{tabular}{l}
0.00 \\
\hdashline$: \infty 00$ \\
\end{tabular} & $\begin{array}{r}6224753 \\
3.042934 \\
\end{array}$ & $\begin{array}{r}8051658 \\
3.480852 \\
\end{array}$ \\
\hline MESz2 <- & $\begin{array}{r}\text { Interpersonal } \\
\text {-cons }\end{array}$ & $\begin{array}{r}3510828 \\
2.750376 \\
\end{array}$ & $\begin{array}{r}1192196 \\
.3776893 \\
\end{array}$ & $\begin{array}{l}7: 14 \\
7: 28 \\
\end{array}$ & \begin{tabular}{l}
0.00 \\
\hdashline$: \infty 00$ \\
\end{tabular} & $\begin{array}{r}6173984 \\
2.010118\end{array}$ & $\begin{array}{r}1.084729 \\
3.490633 \\
\end{array}$ \\
\hline MEs23 <- & $\begin{array}{r}\text { Interpersonal } \\
\text {-cons }\end{array}$ & $\begin{array}{r}8944537 \\
2.485249 \\
\end{array}$ & $\begin{array}{r}008649 \\
6873756 \\
\end{array}$ & $\begin{array}{r}183.42 \\
28: 44 \\
\end{array}$ & \begin{tabular}{r}
0.00 \\
\hdashline \\
\end{tabular} & $\begin{array}{r}877502 \\
2.313996 \\
\end{array}$ & $\begin{array}{r}9114054 \\
2.656502 \\
\end{array}$ \\
\hline MESB $<-$ & $\begin{array}{r}\text { Standing } \\
\text {-cons }\end{array}$ & $\begin{array}{r}7965323 \\
3.741201 \\
\end{array}$ & $\begin{array}{r}0522651 \\
-1208794 \\
\end{array}$ & $\begin{array}{r}15: 24 \\
30: 95 \\
\end{array}$ & 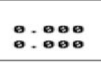 & $\begin{array}{r}6940945 \\
3.504282 \\
\end{array}$ & $\begin{array}{r}8989701 \\
3.998121 \\
\end{array}$ \\
\hline MESI1 <- & $\begin{array}{r}\text { Standing } \\
\text {-cons }\end{array}$ & $\begin{array}{r}6409112 \\
5.099365 \\
\end{array}$ & $\begin{array}{r}0461934 \\
2085231 \\
\end{array}$ & $\begin{array}{l}13: 87 \\
24: 43\end{array}$ & : & $\begin{array}{r}5503738 \\
4.684768 \\
\end{array}$ & $\begin{array}{r}7314485 \\
5.502163 \\
\end{array}$ \\
\hline MESS <- & $\begin{array}{r}\text { Empathetict } \\
\text {-cons }\end{array}$ & $\begin{array}{r}5926628 \\
3.878794 \\
\end{array}$ & $\begin{array}{r}0398425 \\
2197388 \\
\end{array}$ & $\begin{array}{l}14.88 \\
17.65 \\
\end{array}$ & 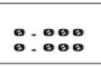 & $\begin{array}{r}5145728 \\
3.448114 \\
\end{array}$ & $\begin{array}{r}6707527 \\
4.309475 \\
\end{array}$ \\
\hline MEsic <- & $\begin{array}{r}\text { Empathetsct } \\
- \text {-cons }\end{array}$ & $\begin{array}{r}-7512866 \\
759607 \\
\end{array}$ & $\begin{array}{r}038087 \\
.3785459 \\
\end{array}$ & $\begin{array}{r}-19: 73 \\
20: 33 \\
\end{array}$ & \begin{tabular}{l}
0.00 \\
\hdashline$: 080$ \\
\end{tabular} & $\begin{array}{r}-.8259357 \\
6.954133 \\
\end{array}$ & $\begin{array}{r}-.6766376 \\
8.438306 \\
\end{array}$ \\
\hline MES17 <- & $\begin{array}{r}\text { Empathetict } \\
\text {-cons }\end{array}$ & $\begin{array}{r}2368595 \\
2.694175 \\
\end{array}$ & $\begin{array}{r}0395081 \\
1082594 \\
\end{array}$ & $\begin{array}{l}22.45 \\
24: 89\end{array}$ & 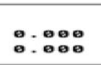 & $\begin{array}{r}8094251 \\
2.48199 \\
\end{array}$ & $\begin{array}{r}9642939 \\
2.908359 \\
\end{array}$ \\
\hline & 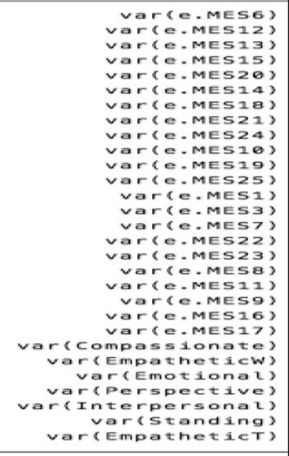 & 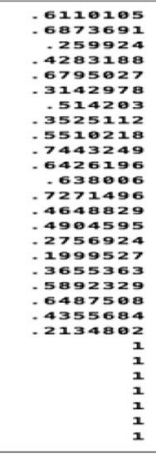 & 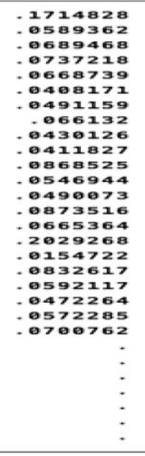 & & & 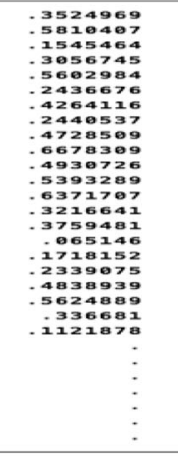 & 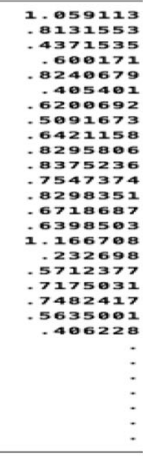 \\
\hline 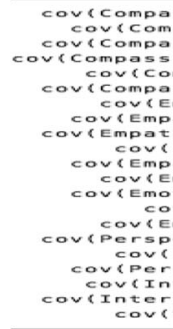 & 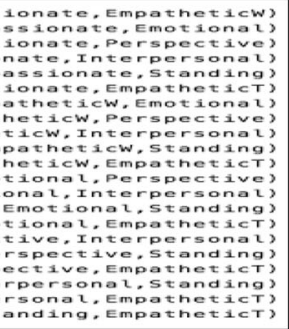 & 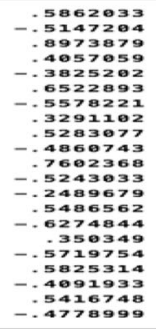 & 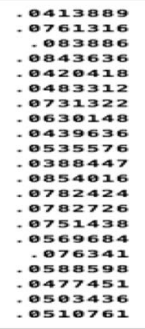 & 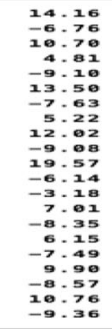 & 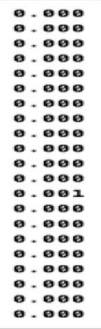 & 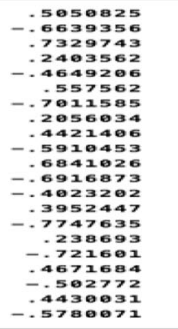 & 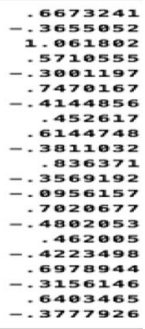 \\
\hline
\end{tabular}

Fig. 3 Estimates, standard error, t-values, error terms and $r 2$ for items of MES 


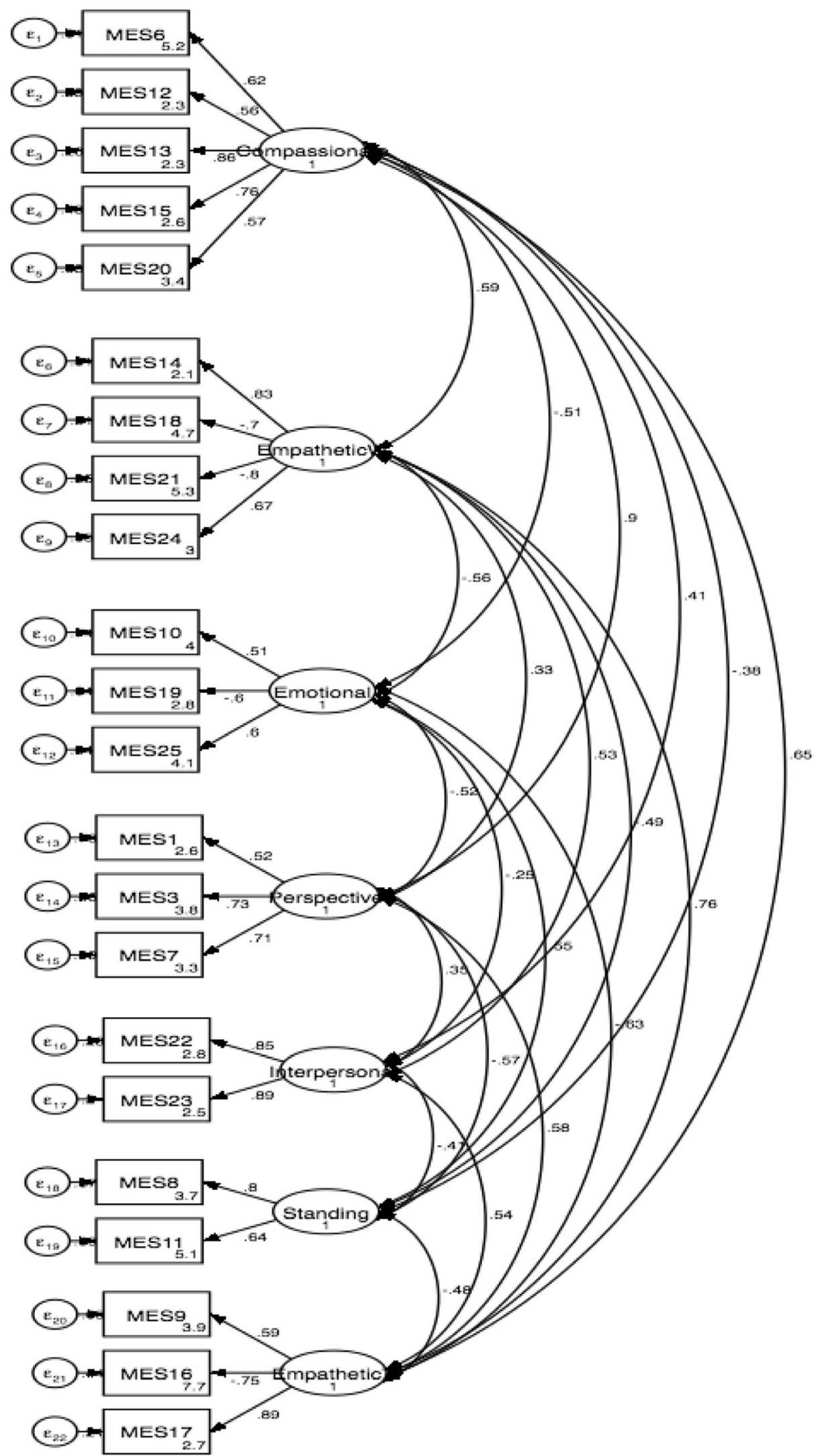

Fig. 4 Confirmatory Factor Analysis for the Midwifery Empathy Scale (MES 
Detachment 4) Perspective Taking 5) Interpersonal Relationship 6) Standing in the Woman's Shoes 7) Empathetic Tendencies. According to MES validation study, 3 out of 25 items were excluded from the analysis, which were not relevant for our sample.

A Barlett's test of sphericity with $(p<0.0001)$ and Kaiser-Meyer-Olkin (KMO) measure of simplicity adequacy of 0.817 were used in performing this factor analysis. A factor was considered important if its eigenvalue exceeded 1 (Kaiser 1960). As factor analysis found 7 independent subscales, subsequent Cronbach's alpha were separately carried out for each subscale, to highlight how the items group together. According to factor analysis 7 subscales have been revealed within the MES. Cronbach's alpha was 0.731 for the first subscale, 0.577 for the second, 0.505 for the third, 0.592 for the fourth, 0.767 for the fifth, 0.515 for the sixth and 0.572 for the seventh.

Our findings confirm the multidimensionality of the MES, demonstrating a seven-factor structure, while the sub-scales of the Greek MES showed very good values for Cronbach's alpha. Significant differences in itemfactor loadings characteristics may be explained by the varied cultural backgrounds of our study population. The confirmatory factor analysis demonstrated that the seven factor model based on the exploratory factor analysis offered a very good fit to the our data. All goodness of fit statistics were found to be very good.

\section{Limitations}

This study was not without limitations. The MES was pilot-tested and validated using samples of midwives and student midwives from the two largest public maternity hospitals in Athens and fewer midwives who work in the private sector where included and independent midwives were not included at all. This sample may not be representative of the population of midwives in Greece and the majority of our subjects were female. Another limitation of the research concerns the fact that the recruited midwives work in a hospital setting (public, private) and there were time availability issues in regards to completion of the questionnaires. For similar reasons as well as stress related factors colleagues of the Central Delivery Units were not invited to participate to the study. Finally, the authors didn't use patient assessments of their midwife's empathy, such as Stewart Mercer's CARE (consultation and relational empathy) scale, a well-validated and reliable measure, as a complementary method of assessing the empathy of midwives that participated to the study $[20,21]$.

\section{Conclusion}

The MES studied in a sample of 242 student and practicing midwives, showed satisfactory reliability and factor analysis indicated seven components. Therefore, we assert that this validated scale may be reliably used for identifying the levels of empathy of student and professional midwives, in order to improve the quality of midwifery care.

\section{Abbreviations}

KMO: Kaiser-Meyer-Olkin; MES: Midwifery empathy scale

\section{Acknowledgements}

We are grateful to all the midwives and student midwives who contributed with their valuable perspectives and time. We would also like to acknowledge the contributions by Prof Christos Lionis, Prof Katerina Lykeridou and Antigoni Sarantaki for the fruitful discussions about measuring the levels of empathy in midwifery clinical practice.

\section{Funding}

The authors declare that they have no funding for the research reported.

\section{Availability of data and materials}

The materials described in the manuscript are readily reproducible, including database and all relevant data. The Midwifery Empathy Scale is freely available to any scientist willing to use it. Software, databases, MES as described in the manuscript are available for testing by reviewers in a way that preserves the reviewers anonymity. Authors wish to share MES and could email the pdf version to any scientist who will contact the corresponding author.

\section{Authors' contributions}

All authors have made substantial contributions to this study; W, PF, AC, PG and NT were responsible for the study design. PF and AC collected the data, $W$ was responsible for the initial data analysis and the writing of manuscript. All authors took part in the interpretation of data and drafting of the manuscript. All authors critically revised, read and approved the final manuscript.

\section{Competing interests}

The authors declare that they have no competing interests.

\section{Consent for publication}

Written informed consent and consent for publication was provided by the participants of the study.

\section{Ethics approval and consent to participate}

Ethical approvals by the Ethical Committees of the Hospitals (No51/27-2-2015 \& No17/24-2-2015) were obtained.

\section{Author details}

${ }^{1}$ Department of Midwifery, Technological Educational Institute of Athens, Athens, Greece. ${ }^{2}$ University of Pennsylvania, Philadelphia, USA. ${ }^{3}$ Department of Midwifery, ATEI of Thessaloniki, Sindos, Greece. ${ }^{4}$ Department of Nursing, TEl Crete, Heraklion, Greece. ${ }^{5}$ Kings's College, London, UK.

Received: 1 June 2016 Accepted: 27 October 2016

Published online: 17 November 2016

\section{References}

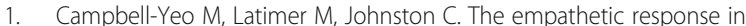
nurses who treat pain: concept analysis. J Adv Nurs. 2007;61(6):711-9.

2. Neumann M, Bensing J, Mercer S, Ernstmann N, Ommen O, Pfaff H. Analyzing the "nature" and "specific effectiveness" of clinical empathy: A theoretical overview and contribution towards a theory-based research agenda. Patient Educ Couns. 2009;74:339-46.

3. Matthew DA. A perspective on cultivating clinical empathy. Complement Ther Clin Pract. 2009;15:76-9.

4. Tarkka M-T, Paunonen M, Laippala P. Importance of the midwife in the firsttime Mother's experience of Childbirth. Scand J Caring Sci. 2000;14:184-90.

5. Hodnett E, Osborn R. Effects of continuous intra-partum professional support on childbirth outcomes. Res Nurs Health. 1989:12:259-97.

6. Tarkka M-T, Paunonen M. Social support and its impact on mothers' experiences of childbirth. J Adv Nurs. 1996;23:70-5.

7. Pascoe JM, French J. The reliability and validity of the maternal social support index for primiparous mothers : a brief report. Fam Med. 1989;22:228-30. 
8. McKenna L, Boyle M, Brown T, Williams B, Molloy A, Lewis B, Molloy L. Levels of empathy in undergraduate midwifery students: An Australian cross-sectional study. Women Birth. 2011;24(2):80-4.

9. Williams B, Brown T, McKenna L, Palermo C, Morgan P, Nestel D, Brightwell R, Gilbert-Hunt S, Stagnitti K, Olaussen A, Wright C. Student empathy levels across 12 medical and health professions: an international study. J Compliance Health Care. 2015;2:4.

10. Williams B, Brown T, Boyle M, McKenna L, Palermo C, Etherington J. Levels of empathy in undergraduate emergency health, nursing and midwifery students: a longitudinal study. Adv Med Educ Pract. 2014;5:299-306.

11. Cormack D. The research process in nursing. Oxford: Blackwell Science; 2000.

12. Tabachnick B, Fidell L. Using multivariate statistics. 3rd ed. N.Y.: AddisonWesley- Longman; 2007.

13. Kaiser HF. The application of electronic computers to factor analysis. Educ Psychol Meas. 1960:20:141-51.

14. Hakstian AR, Rogers WD, Cattell RB. The behaviour of numbers factors rules with simulated data. Multivar Behav Res. 1982;17:193-219.

15. Morrison DF. Multivariate statistical methods. 2nd ed. New York: McGraw-Hill; 1976.

16. Joreskog KG, Sorbom D. LISREL VI: analysis of linear structural relationships by maximum likelihood, instrumental variables, and least squares methods. Sweden: Department of Statistics, University of Uppsala; 1986.

17. Anderson JC, Gerbing DW. The effect of sampling error on convergence, improper solutions, and goodness of fit indices for maximum likelihood confirmatory factor analysis. Psychometrika. 1984;49:155-73.

18. Bentler PM, Chou CP. Practical issues in structural equation modelling. Sociol Methods Res. 1987;16:78-117.

19. Marsh HW, Balla JR, MacDonald RP. Goodness of fit indexes in confirmatory factor analysis: the effect of sample size. Psychol Bull. 1988;88:245-58.

20. Mercer SW, Watt GCM, Maxwell M, Heaney DH. The development and preliminary validation of the Consultation and Relational Empathy (CARE) Measure: an empathy-based consultation process measure. Fam Pract. 2004;21(6):699-705.

21. Murphy DJ, Bruce DA, Mercer SW, Eva KW. The reliability of workplace-based assessment in postgraduate medical education and training: a national evaluation in general practice in the United Kingdom. Adv Health Sci Educ. 2009;13:219-32

\section{Submit your next manuscript to BioMed Central and we will help you at every step:}

- We accept pre-submission inquiries

- Our selector tool helps you to find the most relevant journal

- We provide round the clock customer support

- Convenient online submission

- Thorough peer review

- Inclusion in PubMed and all major indexing services

- Maximum visibility for your research

Submit your manuscript at www.biomedcentral.com/submit

) Biomed Central 